\title{
THE PROCESS OF PLASMA CHEMICAL PHOTORESIST FILM ASHING FROM THE SURFACE OF SILICON WAFERS
}

\author{
Siarhei Bordusau*, Siarhei Madveika, Anatolit Dostanko
}

\author{
Belorussian State University of Informatics and Radioelectronics, Minsk, Belarus \\ * corresponding author: bordusov@bsuir.by
}

\begin{abstract}
At present, the research for finding new technical methods of treating materials with plasma, including the development of energy and resource saving technologies for microelectronic manufacturing, is particularly actual.

In order to improve the efficiency of microwave plasma chemical ashing of photoresist films from the surface of silicon wafers a two-stage process of treating was developed. The idea of the developed process is that wafers coated with photoresist are pre-heated by microwave energy. This occurs because the microwave energy initially is not spent on the excitation and maintenance of a microwave discharge but it is absorbed by silicon wafers which have a high tangent of dielectric losses. During the next step after the excitation of the microwave discharge the interaction of oxygen plasma with a pre-heated photoresist films proceeds more intensively.
\end{abstract}

The delay of the start of plasma forming process in the vacuum chamber of a plasmatron with respect to the beginning of microwave energy generation by a magnetron leads to the increase of the total rate of photoresist ashing from the surface of silicon wafers approximately 1.7 times.

The advantage of this method of microwave plasma chemical processing of semi-conductor wafers is the possibility of intensifying the process without changing the design of microwave discharge module and without increasing the input microwave power supplied into the discharge.

KEYwoRDS: microwave plasma, microwave plasmatron, plasma chemical ashing of photoresist.

\section{INTRODUCTION}

At present, in most cases the technology of plasma chemical photoresist ashing from the surface of semiconductor wafers uses high frequency or microwave discharges the main drawback of which is inertia of the process at the beginning of plasma forming process. Ashing of photoresist begins with a delay connected with warming up of the reaction-discharge chamber's construction elements and wafers. The duration of the delay being dependent on the temperature of the reaction-discharge chamber and wafers, and, consequently, on the size and volume occupied by semiconductor wafers $[8]$. The transition of the microelectronic industry to the use of $200 \mathrm{~mm}$ and even larger diameter wafers needs reaction discharge chambers of more than $0.005 \mathrm{~m}^{3}$ that significantly affects the duration of photoresist ashing with plasma. The formation of high volume and large area microwave discharges leads to certain construction difficulties as industrial microwave discharge plasma sources are designed for $2.45 \mathrm{GHz}$ frequency characterized by a small electromagnetic wave length $(12.2 \mathrm{~cm}$ in open space [12]) and a small depth of electromagnetic field's penetration into plasma.

Experiments to ash photoresist from the surface of silicon wafers during batch processing led to finding out a specific character of "loading" effect manifesting itself in partial absorption of the electromagnetic wave power entering a microwave resonator by the material with a high tangent dielectric loss (silicon) [1, 4, 5]. Peculiar features of microwave energy and silicon wafer interaction should be taken into consideration as well as used in developing and their processing in plasma microwave discharge.

The essence of photoresist plasma chemical ashing lies in the interaction of active particles, as a rule, oxygen plasma (atoms, excited molecules, ions, radicals) with photoresist molecules which are organic compounds [10]. The speed of these reactions is determined by the streams of particles onto the wafer and their temperature. Hence, with the increase of reaction discharge chamber's dimensions, quantity and diameter of plasmatron's semiconductor wafers treated in the chamber, it is necessary to increase the power of supplied microwave radiation. Since medium power microwave magnetrons for technological application have maximum power about $0.8 \div 1.5 \mathrm{~kW} \underline{\underline{6}}$, it becomes necessary to create additional stimulating effects on the process of batch microwave plasma chemical treatment of materials.

The methods of controlling the processes of plasma chemical treatment of semiconductor wafers with the use of gas plasma microwave discharge may be conventionally divided into two groups: by changing the modes of treatment (power, frequency and form of electric pulses etc.) and by changing non electrical operation modes (pressure, type of gas, speed 
of gas stream etc.) [3]. These ways are not always efficient enough and since the increase of rate of photoresist ashing from the wafer surface is proportional to the value of sum power supplied to the discharge from various energy sources, stimulating the treatment process with such types of effects as bombarding by inert gas ions, laser radiation, electron flow, thermal heating before the treatment, heating up with microwave radiation etc., is of great interest [9].

Wide use of additional methods of stimulating the processes of plasma treatment processes of materials is hindered by insufficiently developed technology as well as construction differences in plasma chemical equipment.

In order to stimulate the processes of microwave plasma chemical photoresist ashing from the surface of semiconductor wafers we suggest using microwave warming up before the beginning of plasma treatment. While plasma is absent in the reaction discharge chamber during microwave heating, energy is absorbed straight in the volume of the semiconductor material without the mechanism of heat transfer from plasma to wafers. The reaction of plasma chemical photoresist ashing is endothermic, so photoresist previously warmed up during the stage of plasma treatment will be ashed away quicker.

The purpose of the performed research was to study the application of this method in developing highly effective technologies and the possibility of its use in microwave plasma chemical etching processes using the equipment already available at factories.

\section{METHOD OF EXPERIMENT}

The research was carried out on the base of microwave discharge installation with a quartz tunnel-flow reactor $200 \mathrm{~mm}$ in diameter and $320 \mathrm{~mm}$ in length with a volume about $0.009 \mathrm{~m}^{3}$ placed in the center of a resonator. An M-112 microwave magnetron was used for generating electromagnetic radiation of at least $650 \mathrm{~W}$, the efficiency coefficient being equal to $60 \%$. The total measured power consumed by the installation was equal to $1300 \mathrm{~W}$.

Monocrystal silicon wafers, $76 \mathrm{~mm}$ in diameter and $0.3 \mathrm{~mm}$ thick, coated with of $1.4 \pm 0.1 \mu \mathrm{m}$ thick S1813G2SP15 photoresist film, treated in accordance with the standard modes of lithography, were used as objects for treating. During the experiments to study the rate of photoresist ashing the wafers were placed in the reaction discharge chamber in pairs.

The moment of terminating the microwave plasma chemical photoresist ashing from the surface of silicon wafers was controlled with the spectrometer SL 40-2-2048 ISA with $777.96 \mathrm{~nm}$ oxygen line intensity. $\mathrm{O}_{2}$ from oxygen cylinders was used as plasma forming gas.

The temperature of silicon wafers was measured with the help of a pyrometer Testo 830-T1 (an infrared thermometer).

\section{Results AND THEIR DISCUSSION}

In the result of experiments it was found out that the delay of plasma forming process in a plasmatron vacuum chamber with respect to the beginning of microwave energy generated by a magnetron leads to the increased rate of photoresist ashing from the surface of silicon wafers approximately by 1.7 times and reaches $40 \mathrm{~nm} / \mathrm{s}$. It means that the efficiency of plasma ashing of photoresist from the surface of silicon wafers can be increased with two stage method of treatment. The first stage is warming up of a semiconductor wafers with microwave energy, the second is interaction between microwave plasma and warmed up photoresist.

The essence of the developed two-stage method of plasma chemical photorest ashing from the surface of semiconductor wafers lies in a preliminary warming up of photoresist with microwave energy. Initially, microwave energy is not spent on exciting and maintaining microwave discharge but is completely absorbed by silicon wafers with photoresist having high tangent angle of dielectric losses thanks to which they are warmed up. During further interaction of oxygen plasma with preliminarily warmed up photoresist the process of oxidizing destruction of photoresist is more intensive and is accompanied by volatile components of reaction products, which are removed from the reaction chamber with a vacuum pump.

The delay of plasma formation with respect to the beginning of microwave energy generation by a magnetron was performed in the following way. The reaction discharge chamber is pumped out to a sufficient pressure and then is filled with plasma forming gas to reach the pressure one/two times higher than the working pressure at which plasma will not ignite under the influence of microwave energy. Then the magnetron microwave generation is switched on. The wafers being warmed up for a certain time under the influence of microwave energy, the required pressure of plasma forming gas is set, which provides microwave discharge excitation and the microwave plasma chemical treatment process is performed. Semiconductor wafers are kept in plasma till the photoresist is completely removed from their surface.

Figures 1 and 2 show the dependencies of full cycle duration (microwave warming up and plasma treatment) and the rate of photoresist ashing from the surface of silicon wafers calculated with respect to the total treatment process duration on the time of plasma formation delay with respect to the beginning of microwave generating by the magnetron. Photoresist was ashed from the surface of two Si wafers.

The presented dependencies show that they have an extreme character. We believe it may be connected with the structural changes going on in the photoresist film under the influence of both the temperature factor [7, 13, and the result of processes caused by microwave field [11. 
$\mathrm{T}, \mathrm{s}$

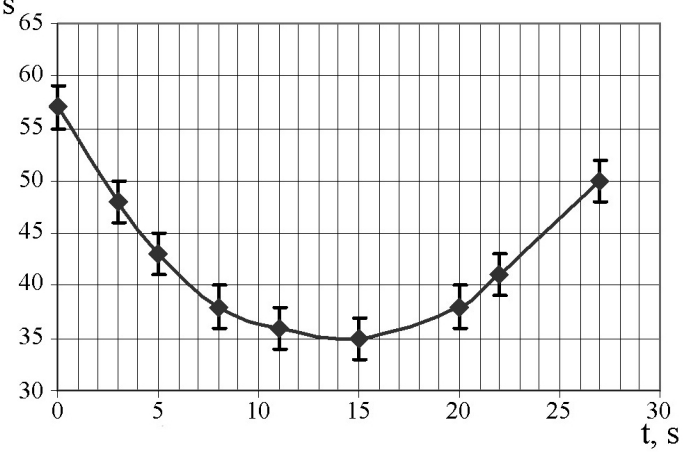

Figure 1. Dependence of total $T$ duration of photoresist ashing from the surface of Si wafers on the delay time $t$ of the beginning of plasma formation with respect to the beginning of microwave energy generation.

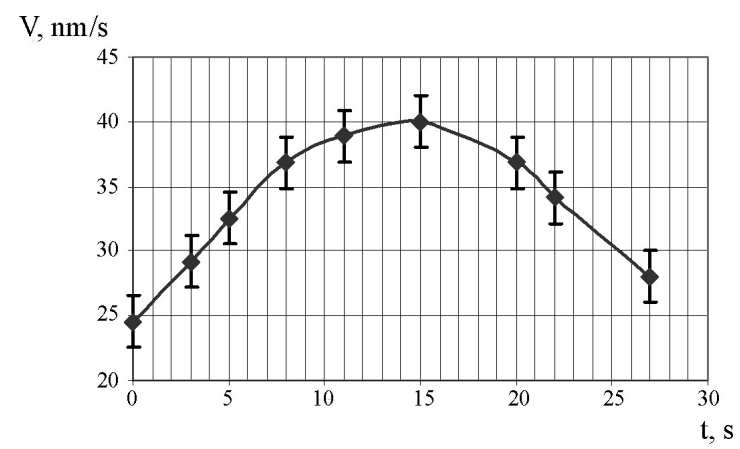

Figure 2. Dependence of the calculated speed $V$ of photoresist ashing from the surface of $\mathrm{Si}$ wafers on the delay time $t$ of the beginning of plasma formation with respect to the beginning of microwave energy generation.

The experiments on the investigation of silicon wafer temperature depending on the time of treating them under the influence of microwave field (Fig. 3) showed, that in the time range of 20 seconds the dependence looks like linear.

According to the data shown in Fig. 3 it is evident that during microwave warming up of silicon wafers their temperature reaches and exceeds the threshold working temperature for this type of photoresist $\left(130 \div 140{ }^{\circ} \mathrm{C}\right)$. Analyzing the data of Figs. 2 and 3 it is possible to make a conclusion that with respect to the conditions of the experiment after $15 \mathrm{~s}$ of microwave energy application, structural changes in the photoresist film may take place. It results in the increase of film resistivity to the process of plasma destruction [2] and, consequently, to the reduction of plasma chemical photoresist ashing rate.

In order to define the rule of revealing the process acceleration effect in case of preliminary microwave warming up of $\mathrm{Si}$ wafers, a series of experiments was carried out to investigate the "loading" effect with reference to the conditions of treatment under investigation. The experiments were carried out loading 2, 5, 10 and 15 wafers into the reaction discharge chamber. The delay of plasma formation with re-
$\Theta,{ }^{\circ} \mathrm{C}$

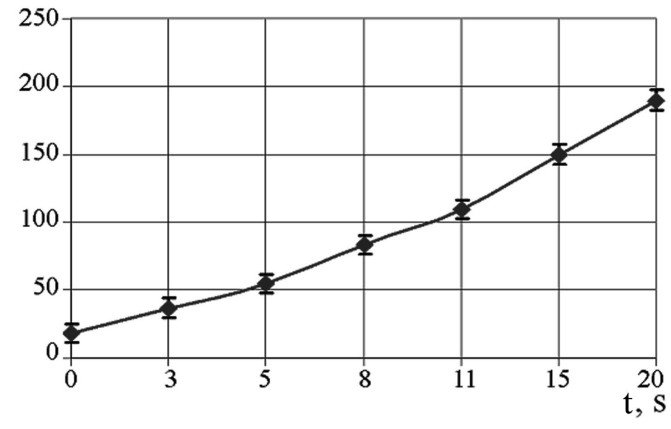

Figure 3. Temperature of Si wafers $\theta$ depending on the time $t$ of their warming up under the influence of microwave energy.

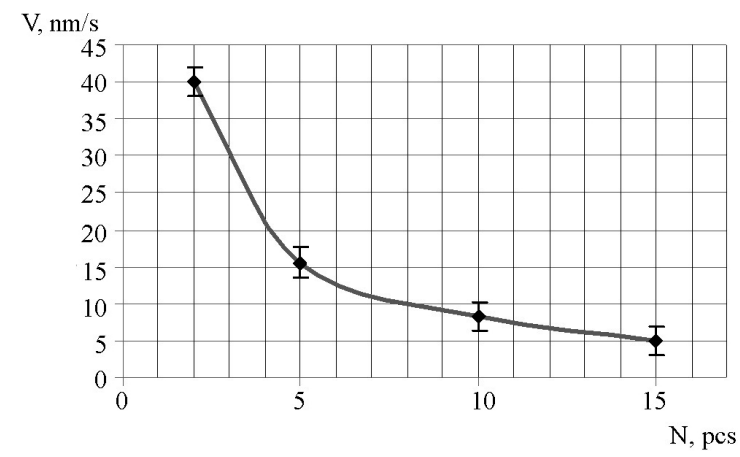

FiguRE 4. Rate of photoresist film ashing from the surface of Si wafers depending on the number of Si wafers in the reactor (the delay duration of the beginning of plasma formation with respect to the beginning of microwave energy generation $t=15 \mathrm{~s}$ ).

spect to the beginning of microwave generation in accordance with the data of Fig. 2 was chosen equal to 15 seconds.

Figure 4 shows the data on the rate of photoresist ashing from the surface of $\mathrm{Si}$ wafers depending on the quantity of $\mathrm{Si}$ wafers in the reaction discharge chamber. Analyzing the obtained data and the data given in [5], we can arrive at the conclusion that the character of "loading" effect in case of two stage treatment is analogous to the classical microwave plasma chemical treatment. We suppose in both cases the process of photoresist film ashing takes place in accordance with the same mechanism but with a higher (up to 1.7 times) rate in case of preliminary activation owing to the microwave warming up.

The experimental data on the investigation of dependence of the rate of plasma chemical photoresist film ashing from the surface of $\mathrm{Si}$ wafers on the pressure of $\mathrm{O}_{2}$ in the reaction discharge chamber is shown in Fig. 5 .

Figure 5 shows that the investigated two-stage process of microwave plasma chemical photoresist film ashing is characterized by the presence of an evident extreme value of photoresist film ashing rate in the range of $\mathrm{O}_{2}$ pressure which qualitatively coincides with the analogous dependence obtained in [5]. 


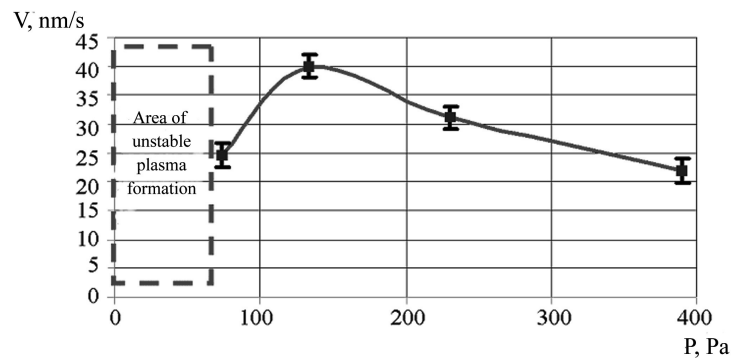

Figure 5. Rate values of photoresist film ashing from the surface of two silicon wafers depending on the pressure $\mathrm{O}_{2}$ (the delay duration of the beginning of plasma formation with respect to the beginning of microwave energy generation $t=15 \mathrm{~s})$.

These results can also confirm the conclusion about the identity of microwave plasma chemical photoresist film coating processes' mechanisms for the studied two-stage method of microwave treatment and a standard single stage process.

\section{Conclusions}

The performed experimental investigation shows that microwave energy can be successfully employed for intensifying plasma chemical processes running at plasma chemical photoresist film coating ashing during the production of electronic devices.

The effect of acceleration the treatment is achieved by absorption of microwave energy in silicon plates and their heating during processing without plasma.

The advantage of such microwave plasma chemical treatment of semiconductor wafers is the possibility of a significant reduction of the process duration without changing the construction of microwave discharge module for technological application and without increasing the microwave power supplied to the discharge.

\section{REFERENCES}

[1] C. Boisse-Laporte, J. Marec (eds.). Microwave Discharges: Fundamentals and Applications. 3rd
International Workshop, Abbaye Royale de Fontevraud, 20 - 25 April, 1997. Les ulis, France, 1998.

[2] S. V. Bordusov. Influence of the microwave plasma processing on the photoresist material. Electronic Materials Processing 5(217):78-80, 2002.

[3] S. V. Bordusov. Microwave plasma technologies in the productions of electronic devices. Bestprint, Minsk, 2002. Edited by A.P. Dostanko.

[4] S. V. Bordusov, S. I. Madveyko. Investigation of the influence of the effect of "loading" the discharge chamber on the optical characteristics of the microwave resonator type plasmatron. Proceedings of Polotsk State University 8:103-106, 2010.

[5] S. V. Bordusov, S. I. Madveyko. Investigation of the influence of electrical regimes of plasma formation on the local chemical activity of the microwave discharge plasma. Proceedings of Polotsk State University 3:119-123, 2012.

[6] A. N. Didenko, B. V. Zverev. Microwave energy. Science, Moscow, 2000.

[7] A. V. Dinaburg, I. G. Erusalimchik, V. S. Zelova, et al. Investigation of physico-chemical properties of positive photoresists. Electronic equipment Sor 2 Semiconductor devices 7(71):17-49, 2009.

[8] V. M. Dolgopolov, V. I. Ivanov, V. A. Krotkov, et al. Spectral control indicator of process removing the photoresist in oxygen plasma. Electronic Engineering Series 7 Technology, production organization and equipment 5(114):27-30, 1982.

[9] A. P. Dostanko, et al. Intensification of solid-state structures production by concentrated energy flows. Bestprint, Minsk, 2005.

[10] A. P. Dostanko, S. P. Kundas, S. V. Bordusov, et al. Plasma processes in the productions of electronic devices. FUAinform, Minsk, 2001.

[11] V. I. Dubkova, S. V. Bordusov, A. P. Dostanko, et al. Investigation of the influence of microwave field on the curing process and properties of epoxy compositions. Journal of Engineering Physics 70(6):1014-1019, 2009.

[12] A. MacDonald. Microwave breakdown in gases. Mir, Moscow, 1969.

[13] W. Moro. Microlithography. Mir, Moscow, 1990. 